\title{
Double origin of hydrothermal convective flux variations in the Fossa of Vulcano (Italy)
}

\author{
Ma urice Aubert • Sere na Diliberto • Anthony Finizola - Yashmin Chébli
}

\begin{abstract}
Soil-temperature measurements can provide information on the distribution of degassing fissures, their relationship to the internal structure of the volcano, and the temporal evolution of the system. At Vulcano Island (Italy), heat flux from a $<3 \mathrm{~km}$-deep magma body drives a hydrothermal system which extends across the main Fossa crater. This heat flux is also associated with variable magmatic gas flow. A high-density map of soil-temperatures was made in 1996 at a constant depth of $30 \mathrm{~cm}$ on the central and southern inner flanks of the Fossa crater. These measurements extended over an area covering about $0.04 \mathrm{~km}^{2}$, across which the heat flux is predominantly associated with a shallow boiling aquifer. The map shows that hot zones relate to structures of higher permeability, mainly associated with a fissure system dating from the last eruptive cycle (1888-1890). From 1996 to January 2005, we studied the evolution of the heat flux for the high temperature part of the map, both by repeating our
\end{abstract}

M. Aubert (凹) · Y. Chébli

Université Blaise Pascal et CNRS-LMV,

5 rue Kessler,

63038 Clermont Cedex, France

e-mail: M.Aubert@,opgc.univ-bpclermont.fr

\section{S. Diliberto}

Istituto Nazionale

di Geofisica e Vulcanologia-Sezione di Palermo,

via Ugo La Malfa, 153,

90146 Palermo, Italy

\section{A. Finizola}

Laboratoire Géosciences Réunion, UR, IPGP, UMR 7154, 15 avenue René Cassin, BP 7151, 97715 Saint Denis cedex 9, La Réunion, France measurements as part of 14 visits, during which temperatures were measured at a constant depth, and using data from permanent stations which allowed soil-temperatures to be continuously measured for selected vertical profiles. These data allowed us to calculate the heat flux, and its variation, with good precision for values lower than about $100 \mathrm{~W} \mathrm{~m}^{-2}$, which is generally the case in the study area. Above $100 \mathrm{~W} \mathrm{~m}^{-2}$, although the heat flux value is underestimated, its variations are recorded with an error less than $10 \%$. During the period 1996-2004, two increases in the thermal flux were recorded. The first one was related to the seismic crisis of November 1998 which opened existing or new fissures. The second, in November 2004, was probably due to magma migration, and was associated with minor seismic activity.

\section{Introduction}

On active volcanoes, the hydrothermal flux towards the surface proves, on one hand, the presence (at depth) of a hot magmatic source, and on the other, the presence of a hot aquifer acting as a thermal buffer between the magmatic heat source and the surface (Montalto 1994). The distribution of this heat flux also depends on the presence and distribution of any fissure systems, and thus on the internal structure of the volcano. Thus, each change in the heat flux (range or distribution) gives an indication of the evolution of the deeper system.

Since the end of the last magmatic eruption (1890), activity at the Fossa crater (Vulcano, Aeolian Island, Italy) has consisted of fumarolic emissions of fluctuating intensity 
(Chiodini et al. 1995). Since 1978, with an earthquake of $\mathrm{M}=5.5$, increased thermal, seismic and geochemical activity has been recorded in the region (Nuccio et al. 1999). Different authors (e.g. Montalto 1996; Italiano et al. 1998; Capasso et al. 1999; Chiodini et al. 2005) have ascribed hydrothermal system variations at the Fossa crater either to changes in regional tectonic factors or to magma migration.

The work presented here focuses on estimating the hydrothermal convective flux, and its variations, using soiltemperature measurements in a subfumarolic area. We relate these variations in this study. This distinction could contribute to the assessment of the risk related to impending eruption and/or seismic activity.

\section{Theoretical aspects of hydrothermal convection}

Figure 1 shows the supposed mixing zone at Vulcano between a hydrothermal system and magmatic gases, and the position of two shallow, boiling, aquifers. Thermal convection above magma bodies is treated by Hardee (1982) as one-dimensional bottom-heated convection process in a permeable medium. Figure 2 summarizes the thermal flux through the different zones above a magma body, from the molten magma up to the surface via a hot aquifer.

Assuming the presence of a heat exchanger, variation in the flux at the surface $(\Phi 2)$ may reflect (1) a variation of the magma-derived flux $(\Phi 1)$ and/or (2) a change in the permeability of the exchanger or unsaturated medium above it (Harris and Maciejewski 2000).

The general relation between fluxes $\Phi 1$ and $\Phi 2$ may be written as (adapted from Aubert 1999):

$$
\Phi 2=\Phi 1 F\left(W_{\mathrm{BP}}, P_{\mathrm{C}}, P_{\mathrm{D}}\right)
$$

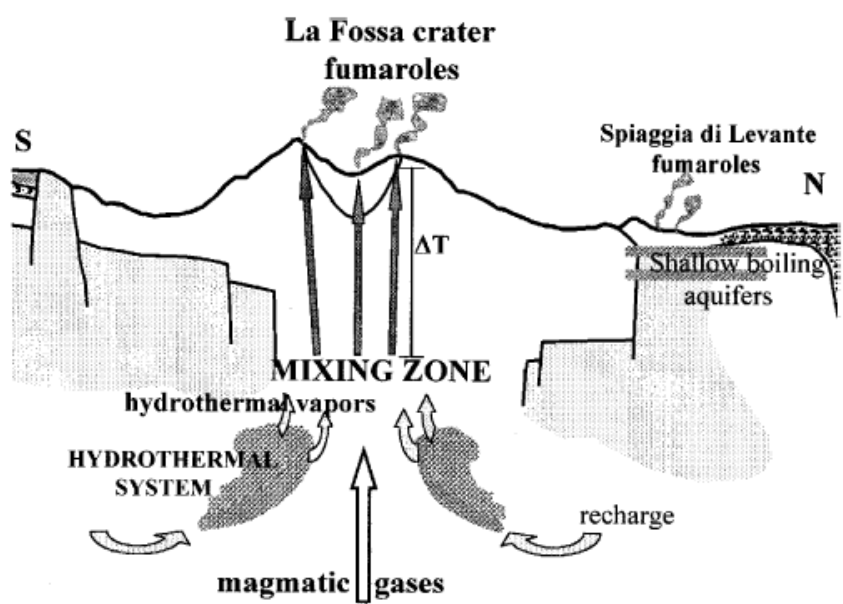

Fig. 1 Mixing process between magmatic gases and hydrothermal vapours within a biphasic hydrothermal system at Vulcano. $\Delta T$ represents the cooling of the fumarolic gases between the mixing zone and the surface. Figure and text from Nuccio et al. (1999)

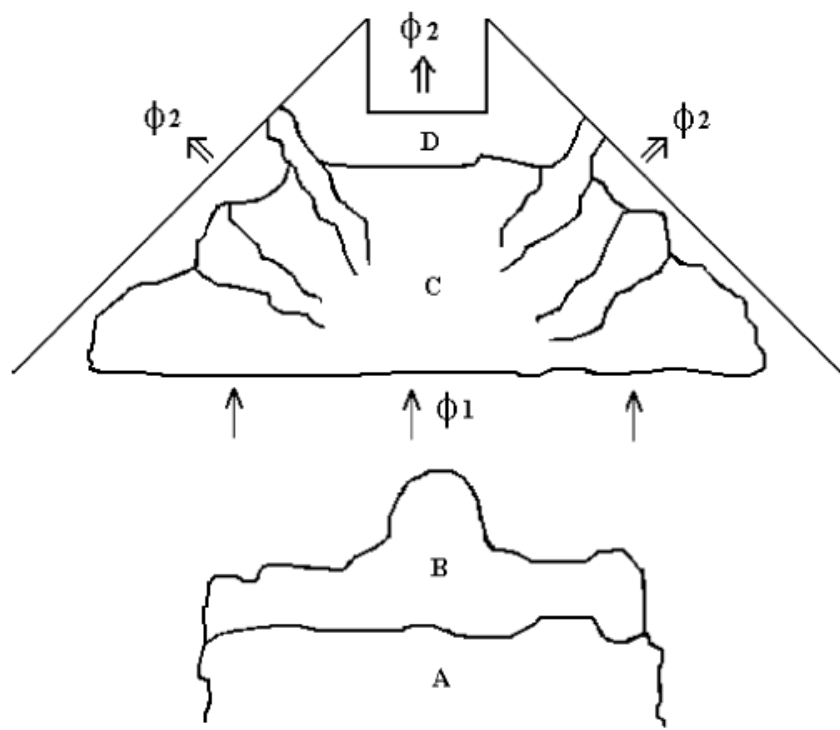

Fig. 2 Thermal zones above magma bodies. a Molten magma body; b crystalline and progressively crystallising intrusion; $\Phi I$ heat flux between magma body and transition zone where conductive flux dominates; $\mathbf{c}$ heat exchanger in aquifer where two-phase permeable convection flux dominates; d unsaturated medium; $\Phi 2$ surface leaving heat flux. From Aubert (1999), which was itself adapted from Hardee (1982) and Harris and Stevenson (1997)

in which, $F$ is a transfer function depending on WBP, i.e. the percentage of bi-phasic water in the unsaturated medium $\mathrm{D}$, as well as $P_{\mathrm{C}}$ and $P_{\mathrm{D}}$, these being the permeabilities of media $\mathrm{C}$ and $\mathrm{D}$ (Fig. 2). Thus, variations in flux $\Phi 2$ may be induced either by changes in $\Phi 1$ or $F$. This effect has often been observed on active volcanoes, including Vulcano (e.g. Aubert and Alparone 2000; Harris and Maciejewski 2000) where the seismo-tectonic activity is considered to play an active role in volcano dynamics (Ventura et al. 1999).

Flux values estimated from soil-temperature measurements

In a porous medium, heat is transferred both by convective and conductive transfer (Aubert 1999). The complete equation to be solved is:

$\delta^{2} T / \delta Z^{2}+Q / \lambda+(\delta T / \delta Z) C_{\mathrm{v}} \rho(V / \lambda)=0$

where $T$ is the soil temperature, $Q$ is the heat quantity produced per unit volume of the medium, $\lambda$ is thermal conductivity, $\rho$ is vapour density, $C_{\mathrm{v}}$ is specific heat of the vapour, $V$ is the vapour ascent velocity, and $\mathrm{Z}$ is depth. For flux values lower than about $100 \mathrm{~W} \mathrm{~m}^{-2}$ the third term of (2) is negligible. Figure 3 gives a theoretical vertical temperature profile from the surface to depth $\mathrm{Z}$ showing three separate zones. From depth $Z 1$ to the surface, $\delta^{2} T / \delta Z^{2}$ equals zero, $Q$ is also zero and the heat transfer is purely 


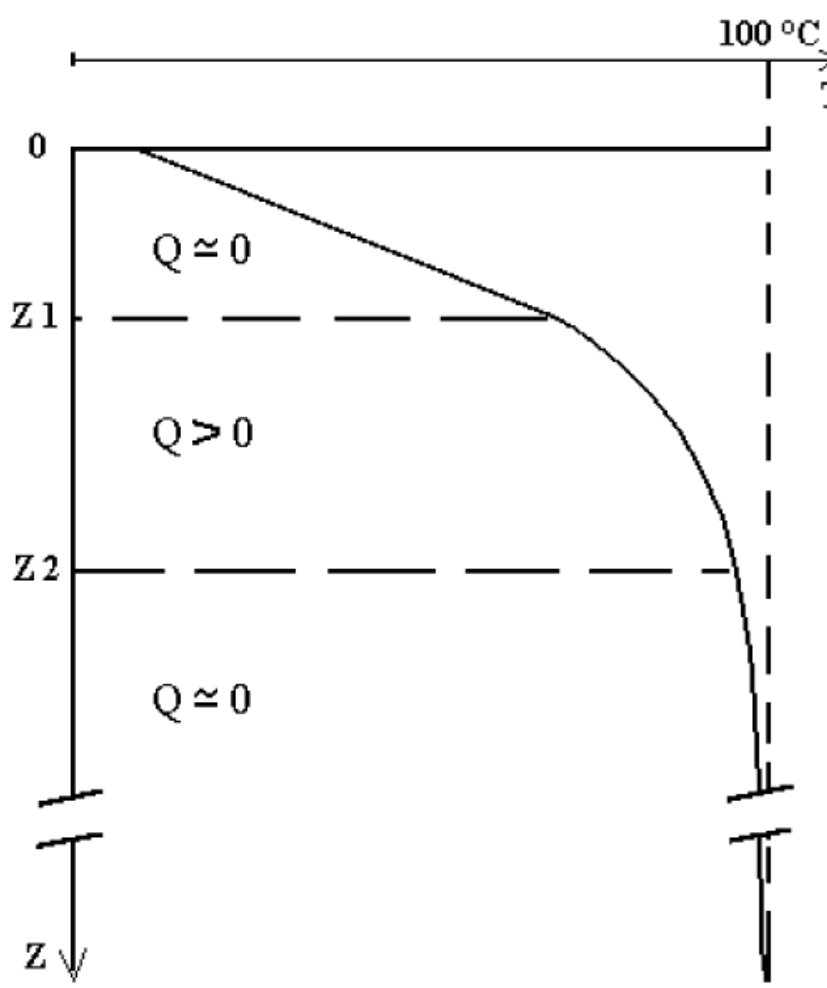

Fig. 3 Theoretical variation in temperature $(T)$ as a function of depth $(Z)$, assuming heat transfer both by conduction and convection (arbitrary units). $Q=$ quantity of latent heat produced by condensation of bi-phase water vapour flow mainly between the depths $Z 1$ and $Z 2$ $(Q>0)$. The conductive transfer is dominant and $\delta T / \delta Z$ constant $(Q \approx 0)$ between $Z 1$ and the surface (from Aubert 1999)

conductive. Across such a conductive dominated zone, heat discharge from the surface can be given by the equation:

$\Phi=\lambda \delta T / \delta Z$

This equation enables $\Phi$ to be calculated when at least three measurements are available along a vertical profile made between depths 0 and $\mathrm{Z1}$ (Fig. 3). If the main objective of measurement is to monitor changes in heat flux with time, it is more convenient to derive only $\delta T / \delta \mathrm{Z}$ because $\lambda$ (thermal conductivity of the rock + liquid phase) is not accurately constrained. When $\Phi$ becomes greater than $100 \mathrm{~W} \mathrm{~m}^{-2} \Phi$ is also equal to the sum of $Q$ from $\mathrm{Z} 2$ to $\mathrm{Z} 1$ (Fig. 3). This can be computed using a Taylor approximation or a B-spline function (Aubert 1999).

The hydrothermal flux carries the thermal power generated at depth by the magmatic source to the surface. This power is, in the case of some active volcanoes such as the Fossa of Vulcano, transmitted by, and partially stored in, a boiling aquifer located between the power source (the magmatic body) and the surface (Fig. 2). Assuming the following characteristics of this aquifer: thickness estimated to a thousand metres (Montalto 1996), ground porosity of $50 \%$, water-vapour density of $1 \mathrm{~kg} \mathrm{~m}^{-3}$, latent heat of condensation of $2.35 \times 10^{6} \mathrm{~J} \mathrm{~kg}^{-1}$, and a saturation degree of
$100 \%$, the latent heat energy density would be very roughly estimated to $1000 \times 0.5 \times 2.35 \times 10^{6}=1 \times 10^{9} \mathrm{~J} \mathrm{~m}^{-2}$. In this way, a limited increase of thermal flux measured at the surface can only provide from the energy stored in the boiling aquifer.

\section{External source variations}

Heat flux measured at the surface of a volcano is dependent upon two terms. The first is the heat generated by sources within the volcano, the second results from the efficiency of the heat exchange through the overlying medium. The latter process is subject to diurnal and seasonal variations. The computation of these variations allows us to:

1. Distinguish external causes of heat flux changes from internal factors

2. Compare instantaneous flux values measured at different times of the day or year

Diurnal and seasonal variations in the air temperature create shallow soil temperature variations that may be calculated. We assume an homogeneous soil with a thermal diffusivity ( $\alpha$, and a sinusoidal wave form to the surface temperature variation $\left(\Delta T_{0}\right)$. At depth $\mathrm{Z}$, the temperature range $\Delta T_{\mathrm{z}}$ is equal to:

$\Delta T_{z}=\Delta T_{0} e^{-Z \sqrt{ } \omega / 2 \alpha}$

where $\omega=2 \pi F$ ( $F$ being the frequency of the wave). If temperature is measured at two depths, $\mathrm{Z} 1$ and $\mathrm{Z} 2$, then $\alpha$ can be calculated from:

$\alpha=(\omega / 2)\left[(Z 2-Z 1) /\left(\ln \Delta T_{Z 1}-\ln \Delta T_{Z 2}\right)\right]^{2}$

The derived value of $\alpha$ is next used to calculate thermal conductivity $\lambda$, using $\alpha \cdot \rho \cdot \mathrm{Cp}$, in which $\rho$ and $\mathrm{Cp}$ are the density and specific heat capacity of the soil. These values can be measured or estimated from mean published values (e.g. Tabbagh and Trezeguet 1987) $\left(\mathrm{Cp}=900 \mathrm{~J} \mathrm{~kg} \mathrm{~K}^{-1}\right.$, $\rho=1,500 \mathrm{~kg} \mathrm{~m}^{-3}$ ). For the Fossa of Vulcano, we calculate $\alpha$ using hourly temperature records at the depths of $30 \mathrm{~cm}$ $\left(T_{30}\right)$ and $10 \mathrm{~cm}\left(T_{10}\right)$ between 15 and 27 August 1998 when $T$ variations were very regular. The mean $\alpha$ value is equal to $6 \pm 0.5 \times 10^{-7} \mathrm{~m}^{2} \mathrm{~s}^{-1}$. This value yields a value for thermal conductivity $\left(\lambda\right.$ of $0.8 \mathrm{~W} \mathrm{~m}^{-1} K^{-1}$, with a relative error of $\pm 30 \%$. When the wave is non-sinusoidal, it is possible to calculate $\alpha$ by using software such as Wave 3.7. (Fortran Scientific Subroutine Library 1985).

Table 1 indicates the theoretical variations in temperature at a depth of $30 \mathrm{~cm}\left(T_{30}\right)$ and $\delta T / \delta Z$ calculated using $\left(T_{30}-T_{10}\right) / 0.2$. These values are calculated using surface temperature $T_{0}$ variations equal to $+/-10^{\circ} \mathrm{C}$, and a mean value of $\alpha$ equal to $6 \times 10^{-7} \mathrm{~m}^{2} \mathrm{~s}^{-1}$. These theoretical values are deduced from temperature measurements taken during 
Table 1 Theoretical annual variation in temperature for the Fossa of Vulcano at a depth of $30 \mathrm{~cm}\left(T_{30}\right)$ and $\delta T / \delta Z\left({ }^{\circ} \mathrm{C} \mathrm{m}^{-1}\right)$ between the depths of $10-30 \mathrm{~cm}$, for a sinusoidal variation in temperature at the surface $\left(T\right.$ range $+/-10^{\circ} \mathrm{C}$; maximum and minimum on 1 January and on 1 July respectively; diffusivity $\alpha=6 \times 10^{-7} \mathrm{~m}^{2} \mathrm{~s}^{-1}$ (from Aubert 1999)

\begin{tabular}{|c|c|c|c|c|c|c|c|c|c|c|c|c|}
\hline & Jan. & Feb. & Mar. & Apr. & May & Jun. & Jul. & Aug. & Sep. & Oct. & Nov. & Dec. \\
\hline$T_{30}\left({ }^{\circ} \mathrm{C}\right)$ & -8.1 & -8.8 & -7 & -3.7 & 0.9 & 5.1 & 8.1 & 8.8 & 7.2 & 3.5 & -0.9 & -5.3 \\
\hline$\delta T / \delta Z\left({ }^{\circ} \mathrm{C} \mathrm{m}^{-1}\right)$ & 6.5 & -8.5 & -18.5 & -27 & -26.5 & -19.5 & -7 & 7.5 & 20.5 & 26.5 & 27 & 19 \\
\hline
\end{tabular}

the period March 2004-September 2005 at a cold (ambient non-geothermally influenced) station (S3) located on the westside of the cone (alt. $40 \mathrm{~m}$ ). The $T_{50}$ (temperature at $50 \mathrm{~cm}$ depth) half-range during this period was between 9 and $10^{\circ} \mathrm{C}$. The relative error calculated for $\alpha(30 \%)$ induces a relative error of less than $4 \%$ for $T_{\mathrm{z}}$ and $\delta T / \delta Z$.

Another external effect on the hydrothermal flux is that of rain which is clearly identified in hourly records because it causes brief (a few tens of days) but intense $\left(\mathrm{a}\right.$ few ${ }^{\circ} \mathrm{C}$ ) cooling. This effect is difficult to model, depending on many local variables, and is considered as noise in our measurements.

\section{Selection of stations to record heat flux variations}

Soil-temperature mapping and selected measurement locations

Since 1997, we have used soil-temperature sensors on the southern inner flank of the Fossa crater to measure the convective flux from the hydrothermal system. Two types of measurement were taken. The first involved five repeated temperature measurements made at the same depth along one profile $T(\mathrm{X})$. Values were then corrected for the external variations following Eq. (4). The second involved three permanent stations (S1, S4 and S5) at which continuous measurements were made at different depths. These sites are located in Fig. 4, where site location was guided by a preliminary soil-temperature mapping.

The soil-temperature mapping was performed in 1996 by measuring soil temperatures along $46 \mathrm{E}-\mathrm{W}$ profiles at a constant depth of $0.3 \mathrm{~m}$. The resulting map reflects the temperature distribution during a period of enhanced energy release from the hydrothermal system (Capasso et al. 1999). The measurement interval was $2.5 \mathrm{~m}$ along each profile, and each profile was separated by $5 \mathrm{~m}$. The resulting soiltemperature map was constructed by interpolation between each measurement point following griding using a $5-\mathrm{m}$ square mesh. Figure 4 shows the three zones (N, C and S) where the measurements were been taken. Collectively they cover a surface area of about $0.04 \mathrm{~km}^{2}$.

The best location for measuring vertical temperature profiles will be near hot zones identified from the mapping but not at the hottest location because here temperature would be buffered at the boiling temperature, about $99^{\circ} \mathrm{C}$ at this altitude. Thus, the repeated profile (AS1S4, Fig. 4) and the permanent stations were located near the thermal anomaly within zone $\mathrm{C}$. The anomaly extends along a line running roughly parallel to the present-day southern crater rim, and is possibly related to a fault system in this location (see the dashed line in Fig. 4). The A-S1-S4 profile intersects the $\mathrm{N}$ side of this anomaly and runs in a direction roughly parallel to the 1888-1890 crater rim. Continuous $T(Z)$ measurements were carried out at three sites: S1, S4 and S5.

As well as the thermal anomaly of zone $C$, the entire mapped area displays many spatial variations in temperature which must be related to the non-homogeneous nature of the sub-surface: cold zones may be related to low permeability zones where vulcanian bombs, mud, and other

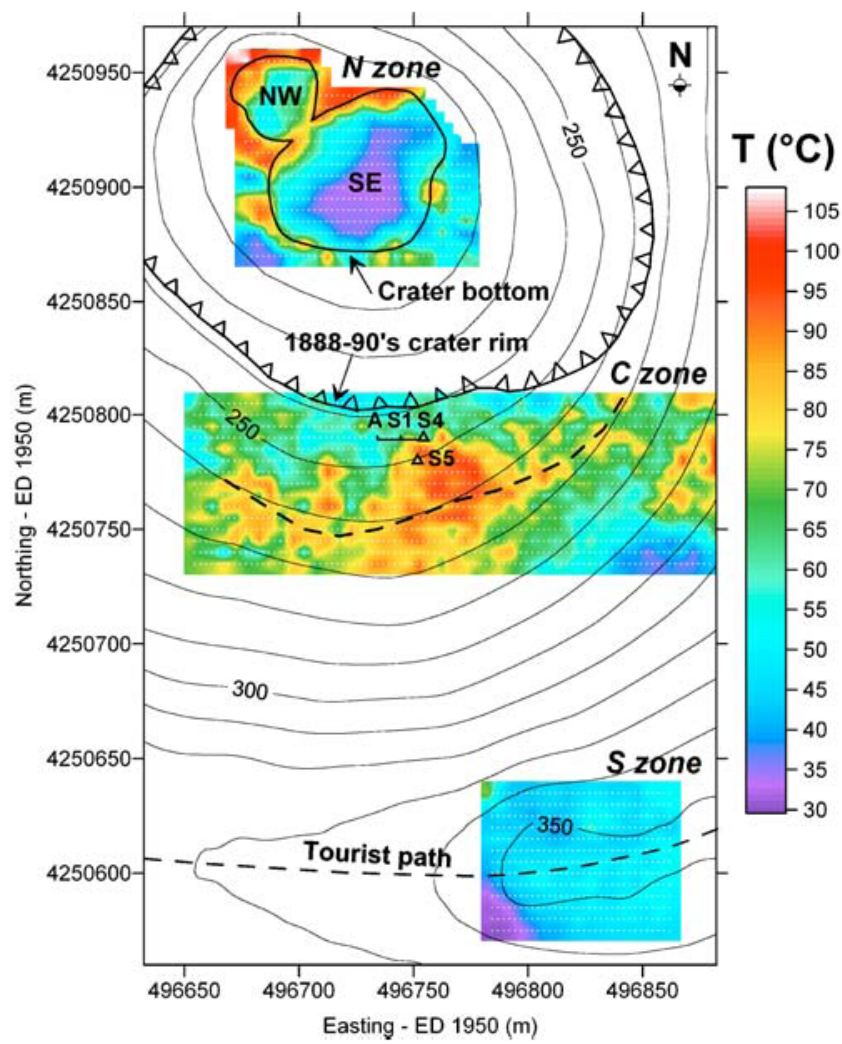

Fig. 4 Map of soil-temperature (T) (measured at a depth of $30 \mathrm{~cm}$ below the surface) across the southern part of the Fossa crater superimposed on volcano-structural features. $A-S I-S 4$ is the profile presented in Fig. 5 (distance AS4=40 m) along which repeated soiltemperature measurements were made at a constant depth of $30 \mathrm{~cm}$. $S 1, S 4$ and $S 5$ (distance $S 4-S 5=18 \mathrm{~m}$ ) are stations where continuous measurements were made along a vertical temperature profile 


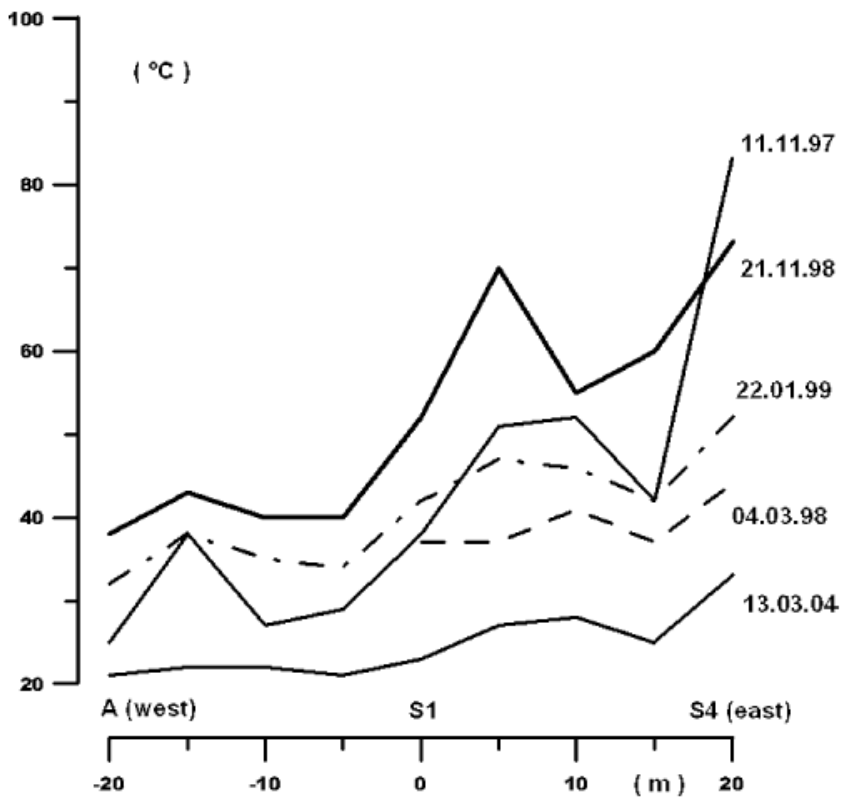

Fig. 5 Evolution of temperature at a constant depth of $30 \mathrm{~cm}$ along the profile A-S1-S4 between November 1997 and 13 March 2004. The distance between two adjacent measurements is $5 \mathrm{~m}$. Values have been corrected for diumal and annual external variations

products may impede the hydrothermal flux. This effect is particularly obvious in the SE crater where the temperatures are uniformly cold (Fig. 4). Rainwater drains down into the bottom of the crater where fine sediments collect to entirely obstruct the hydrothermal convective flux.

\section{Procedure}

\section{Temporary deployments}

The thermistors that we used for temporary measurements had a resolution at $0.1^{\circ} \mathrm{C}$, an accuracy of about $0.5^{\circ} \mathrm{C}$ and a time stability of the order of $1^{\circ} \mathrm{C}$ for a year. Equilibrium between soil temperature and sensor temperature was reached after around $15 \mathrm{~min}$. The sensor was installed in at a depth of $0.3 \mathrm{~m}$ where the diurnal variation is always less than $0.5^{\circ} \mathrm{C}$. Values shown in Fig. 5 are corrected for the annual variations given in Table 1 .

\section{Continuous records}

The first continuous measurement period began in November 1997, and involved a vertical temperature profile at S1 and involved measurements at seven depths: $5,15,25,35$, 45,55 and $65 \mathrm{~cm}$. Many failures occurred and the continuously available record only covers the period between 20 June and 19 September 1998 so that periodic measurements were also made at this site until January 1999 (Fig. 6a).

Beginning on 13 March 2004, continuous measurements were made using new equipment installed at two stations (S4 and S5) inside the Fossa crater (Fig. 4). They consisted of a four-channel datalogger recording data from four temperature sensors at depths of $10,30,50$ and $70 \mathrm{~cm}$. Between March and October 2004, the datalogger was buried in the soil. However, due to overheating of the battery, some data were lost between May and September. After October 2004 the datalogger was kept in an aerated case about $30 \mathrm{~cm}$ above ground. This new arrangement allowed us to register the ensuing anomalous period of increased heat flux.

For flux values less than about $100 \mathrm{~W} \mathrm{~m}^{-2}$, the four chosen depths allow us to make at least three measurements across the linear section of the temperature profile covering section $0-\mathrm{Z} 1$ on Fig. 3. At S5 where the condition $\Phi \leq 100 \mathrm{Wm}^{-2}$ was generally not fulfilled $\left(\mathrm{d} T / \mathrm{d} Z \leq 125^{\circ} \mathrm{C}\right.$ $\mathrm{m}^{-1}$ ) the flux values are often underestimated.
Fig. 6 Two cases of hydrothermal flux variations. a Seismic crisis in November 1998. Continuous and discrete measurements of $\mathrm{d} T / \mathrm{d} Z$ at station $\mathrm{S} 1$. Time interval is about $50 \mathrm{~h}$ between the last shock and the following peak in the hydrothermal flux measurement. b Magmatic crisis in November 2004. Continuous measurement of $\mathrm{d} T / \mathrm{d} Z$ at $\mathrm{S} 4$ (time axis for $\mathrm{X} 2$ enlarged by 3 compared to $\mathrm{X} 1$ )

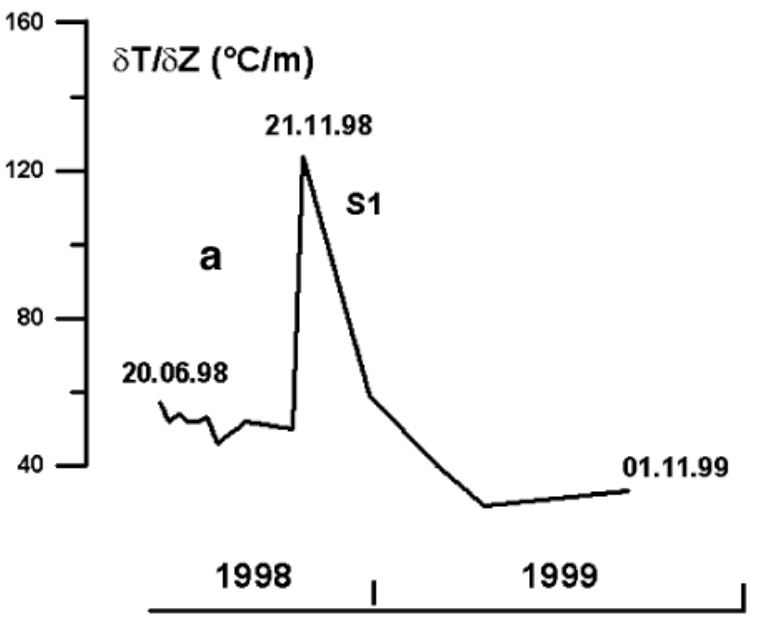

$\mathrm{X} 1$

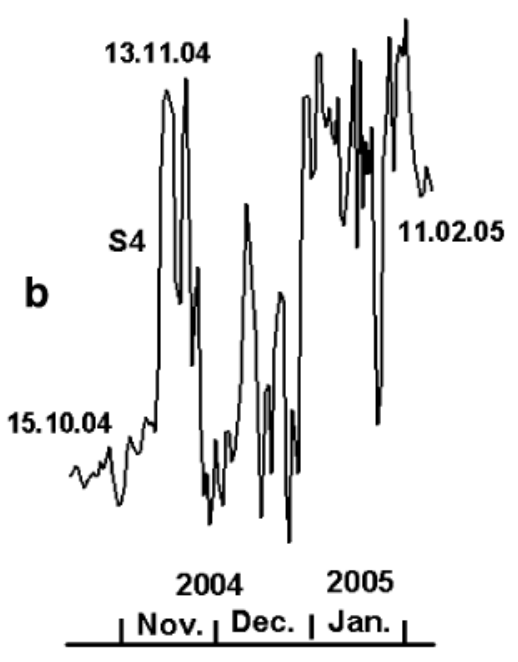

$\times 2$ 


\section{Results}

Temporary deployment results 1997-2004

From 11 November 1997 to 13 March 2004, five temperature profile measurements were taken at S2 and along the A-S1-S4 profile (Fig. 4), which cuts the boundary of the thermal anomaly moving from cold in the west (station A) to hot in the east (station S4). While Fig. 5 indicates the spatial and temporal evolution of $T_{30}$ along this profile, Fig. 8 charts the temporal evolution of $T_{30}$ at $\mathrm{S} 4$ alone. These records show four main results:

1. Spatial variability in the temperature values along each profile is evident (Fig. 5). This is strongly related to the size of the fall-out material of the last eruptions. This material comprises dense bombs, ranging from fine particles to blocks reaching $10 \mathrm{~m}$ across.

2. The general trend along the profile is relatively stable over this 7-year period. In particular, the boundary between the cold and hot zones remains in a constant location, about $5 \mathrm{~m}$ to the west and $\mathrm{NW}$ of $\mathrm{S} 1$. This indicates the presence and persistence of the fissure system in this area.

3. With the exception of our November 1998 data point, all values show a decrease between November 1997 and March 2004. The high values of 1997 are no doubt the continuation of the high values mapped in August 1996 (Fig. 4). This variation is consistent with temporal and spatial thermal evolution recorded for the fumarole field in the northern rim of the Fossa crater between 1994 and 1999 (Harris and Maciejewski 2000).

4. The high values obtained on 21 November 1998 follow the seismic event of 19 November 1998. At this point, temperatures increased at all sites along the profile, with temperatures being highest at the hotter (eastern) side. The seismic energy unsettled the entire measurement zone, but especially those parts which were already fissured.

$T_{30}$ measurements at $\mathrm{S} 4$ provide the most continuous data set for the period 1996 through 2004. Figure 8 confirms that, at this station, apart from November 1998 event, $T_{30}$ declined over this period. However, during 2004, $T_{30}$ values increased once more, returning to the same high values of August 1996. Figure 8 indicates a correlation with $\mathrm{CO}_{2}$ concentration measurements made at the fumarolic field (north side of the crater) by Granieri et al. (2006) as well as from unpublished data of the INGV section of Palermo.

Continuous measurements I: periods of little change

Except for the event of November 1998, the daily mean values of $\delta T / \delta Z$ at S1 show near constant values of around $50^{\circ} \mathrm{C} \mathrm{m}^{-1}$ (Fig. 6a), corresponding to $40 \pm 10 \mathrm{~W} \mathrm{~m}^{-2}$. The short period variations recorded from June to September 1998 are probably caused by external factors (particularly by rainfall). The range of these variations (less than $10^{\circ} \mathrm{C}$ $\mathrm{m}^{-1}$ ) constitutes noise added to the thermal signal related to internal sources. No tectonic seismicity was recorded during this period. At the stations $\mathrm{S} 3, \mathrm{~S} 4$ and $\mathrm{S} 5, \delta T / \delta Z$ was relatively steady between March and October 2004 at about 0,40 and $140^{\circ} \mathrm{C} \mathrm{m}^{-1}$ at the three stations respectively. Only two events are clearly recorded from June 1998 to November 2004. There are described in the following.

Continuous measurements II: periods of large change

\section{Transitory period of November 1998}

The November 1998 thermal and seismic crisis was described in detail by Aubert and Alparone (2000). The seismic crisis comprised five tectonic shocks that were recorded between 17 and 19 November, the magnitudes of which ranged from 1.3 to 2.4 . They were typical faulting events, with clear P-phases and detectable S-phases. The identification of these phases and the spectral analysis of the signals suggest a mechanism of shear fracturing of moderately competent rocks (Montalto 1994). The maximum length of the active fault was estimated at $320 \mathrm{~m}$ (Montalto 1994). The focal mechanism and the moment are, unfortunately, unknown.

During the morning of 21 November, $\delta T / \delta Z$ at $\mathrm{S} 1$, located at more than $1 \mathrm{~km}$ from the earthquake epicenter, reached a maximum value of $124^{\circ} \mathrm{C} \mathrm{m}^{-1}$, greatly exceeding the normal value of about $50^{\circ} \mathrm{C} \mathrm{m}^{-1}$ (Fig. 6a). The rate of increase in $\delta T / \delta Z$ cannot be calculated because of the lack of measurements between the time of the shock and the next temperature profile measurement on 21 November. However, the resulting increase $\mathrm{d} T / \mathrm{d} Z$ is the only clearly observed change in our time series between November 1997 and February 2005. In contrast, the cold part of the profile was only slightly affected by the shock (Fig. 5). The increase in flux at $\mathrm{S} 1$ represents about $30 \mathrm{~W} \mathrm{~m}^{-2}$ over 3 months $\left(7.8 \times 10^{6} \mathrm{~s}\right)$, that is a thermal energy by $\mathrm{m}^{2}$ of about $30 \times 7.8 \times 10^{6}=2.34 \times 10^{8} \mathrm{~J} \mathrm{~m}^{-2}$.

This value is about a quarter of the one roughly estimated energy value stored in the aquifer. Thus, this seismic event could probably not have caused significant change in the thermo-dynamic state near the magmatic source.

Different authors have described the volcano-earthquake interaction (e.g. Hill et al. 2002; Walter and Amelung 2006), showing that both the static and dynamic stress changes may have a triggering effect on volcanic activity. In order to explain the large and rapid variation of heat flux that occurred in November 1998 at the Fossa of Vulcano, we assume that the earthquake induced micro-fissure 

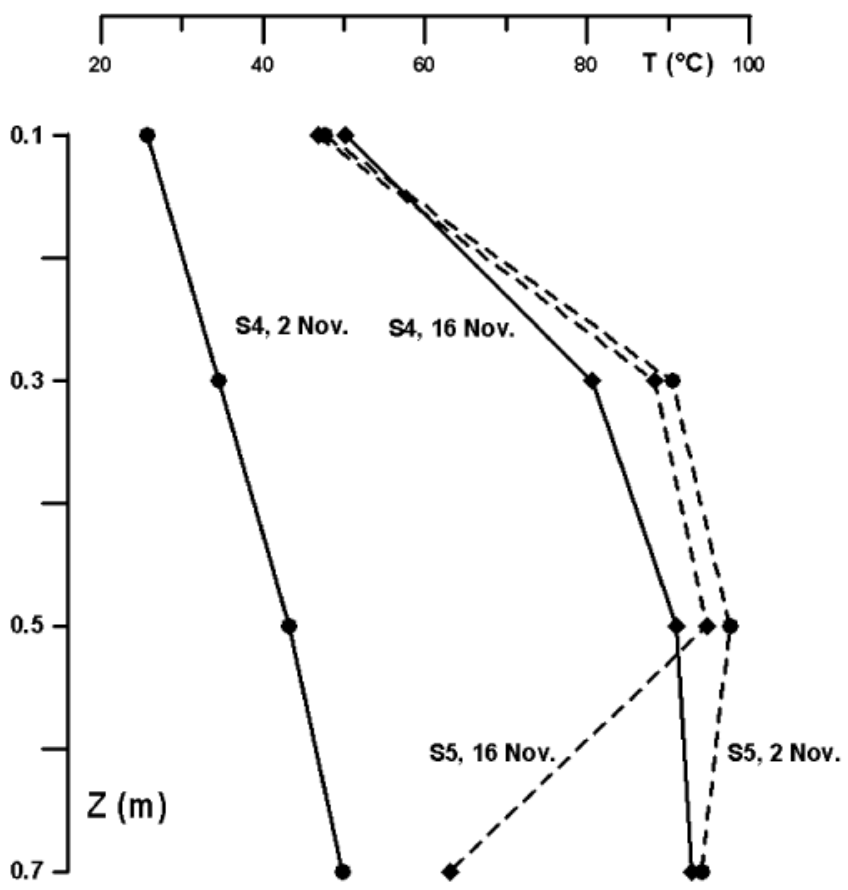

Fig. 7 Temperature profiles taken at S4 (solid line) and S5 (broken line) for the 2 and 16 November 2004 (circles and diamonds respectively)

reopening in areas where the fissure system was already present. This reopening would allow a larger flux of vapour + heat to ascend to the surface. The subsequent decline of the heat fluxes to values typical of the pre-earthquake levels within 4 months indicates that the perturbation was short-lived.

Another possible cause of increased heat would be from the frictional heat produced during the seismic crisis by sliding along the fault plane (McKenzie and Brune 1972). It was not possible to calculate the focal mechanism, and thus we were unable to make a hypothesis regarding the likely values of such thermal and mechanical energies. However, such an input seems unlikely considering the fault length $(<320 \mathrm{~m})$, the low earthquake magnitude (2.4) and the distance between $\mathrm{S} 1$ and seismic foci $(>1 \mathrm{~km})$.

\section{Transitory period of November 2004}

During November 2004, a large change occurred in $\delta T / \delta Z$ at stations S4 and S5. The following discussion focuses on the results from the station $\mathrm{S} 4$ for which data are more continuous. Figure $6 \mathrm{~b}$ indicates the variation in $\delta T / \delta Z$ at $\mathrm{S} 4$ between 15 October 2004 and 11 February 2005, the most obvious variation occurring during the night of 13-14 November 2004 , when $\delta T / \delta Z$ suddenly increased by a factor of 3. Figure 7 shows how temperatures increased at S4 and S5 between 2 and 16 November 2004. The high flux value recorded at S4 on 16 November (about $120 \mathrm{~W}$ $\mathrm{m}^{-2}$ ) corresponds to the upper limit for which Eq. (4) is still applicable. It is not the case at S5 on 2 and 16 November for which only Eq. (2) can be applied. The lack of data, however, means that this equation cannot be solved and thus the flux values for S5 are underestimated. For example, the flux value calculated at S5 for 16 November is about $170 \mathrm{~W} \mathrm{~m}^{-2}$ via Eq. (4), about $200 \mathrm{~W} \mathrm{~m}^{-2}$ via an equation of the third degree.

Table 2 indicates the roughly estimated energy density $\left(7.7 \times 10^{8} \mathrm{~J} \mathrm{~m}^{-2}\right)$ released at $\mathrm{S} 4$ during four months between 15 October 2004 and 11 February 2005. This energy is three times that estimated for the event of November 1998. Table 3 gives the mean and standard deviation for $\delta T / \delta Z$ at stations S4 and S5 during two 1-month-long periods characteristic of quiet (25 March-23 April 2004) and moderate (18 October-16 November 2004) activity. It underlines three points:

1. The average value of heat flux was about five times higher at S5 than S4 during the quiet period of 25 March-23 April 2004), but the increase between the quiet period and moderate periods (18 October-16 November 2004) was less at S5 than at S4 because the high values at $\mathrm{S} 5$ were underestimated

2. The standard deviations are more or less proportional to the average values for S4 and S5 during the quiet period. We assume that the variability of the flux calculated between 0.1 and $0.3 \mathrm{~m}$ is at least partially related to the process of condensation of vapour creating the heat source $Q$ in Eq. (2), but the heat flux at greater depth could stabilize through time. This variability increases between the quiet and moderate periods, even more at S4 where calculations were considered as exact. The ratio of standard deviation versus average value for S4 increases from 0.16 to 0.57 . We assume that this result indicates an increase in the heat source itself.

3. Data retrieved from stations S4 and S5, on the day they were installed, were of good quality. On this date, $\delta T /$

Table 2 Mean fluxes for station S4 between the 15 October 2004 and 11 February 2005

\begin{tabular}{llllll}
\hline Date range & $15.10 .04-13.11 .04$ & $14.11 .04-28.11 .04$ & $29.11 .04-29.12 .04$ & $30.11 .04-11.02 .05$ & Total time \\
\hline Duration (days) & 30 & 13 & 33 & 44 & 120 \\
Mean $\delta T / \delta Z\left({ }^{\circ} \mathrm{C} \mathrm{m}^{-1}\right)$ & 51 & 124 & 64 & 135 & 93 \\
Mean flux $\left(\mathrm{W} \mathrm{m} \mathrm{m}^{-2}\right)$ & 40 & 100 & 50 & 110 & 74 \\
Energy density $\left(\times 10^{8} \mathrm{~J} \mathrm{~m}^{-2}\right)$ & 1 & 1 & 1.4 & 4 & 7.7 \\
\hline
\end{tabular}


Table 3 Mean and standard deviation of $\delta T / \delta Z$ at stations S4 and S5 during two periods relating to quiet activity and moderate thermal activity

\begin{tabular}{lllll}
\hline & $\begin{array}{l}25.03 .04 \\
\mathrm{~h}-23.0404\end{array}$ & $\begin{array}{l}18.10 .04- \\
6.11 .04\end{array}$ \\
\hline$\delta T / \delta Z$ & Mean & $\begin{array}{c}\text { Standard } \\
\text { deviation }\end{array}$ & Mean & $\begin{array}{c}\text { Standard } \\
\text { deviation }\end{array}$ \\
$\left({ }^{\circ} \mathrm{C} \mathrm{m}^{-1}\right)$ & & 5 & 56 & 32 \\
$\mathrm{~S} 4$ & 31 & 5 & 182 & 43 \\
$\mathrm{~S} 5$ & 137 & 19 & 3.2 & 1.3 \\
$\mathrm{~S} 5 / \mathrm{S} 4$ & 5 & 3.8 & & \\
\hline
\end{tabular}

$\delta Z$ was equal to 32 at $\mathrm{S} 4$ and $121^{\circ} \mathrm{C} \mathrm{m}^{-1}$ at $\mathrm{S} 5$, with $T_{70}$ being 40 at $\mathrm{S} 4$ and $87^{\circ} \mathrm{C}$ at $\mathrm{S} 5$. If the flux had decreased after this date, data from station $\mathrm{S} 5$ could be useful to calculate $\delta T / \delta Z$ variations even when $\delta T / \delta Z$ values at station $\mathrm{S} 4$ could become very low. However, the mean $T_{70}$ values at $\mathrm{S} 5$ remained around $90^{\circ} \mathrm{C}$ for the two periods, while mean $T_{70}$ values at $\mathrm{S} 4$ increased from 40 to $56^{\circ} \mathrm{C}$.

\section{Discussion and conclusion}

We identify two possible causes for observed changes in the hydrothermal flux at the Fossa of Vulcano during 19982005: (1) seismic factors, and (2) magma migration. The temporal variation of the flux leads us to believe that the permeability of the superficial layer as well as the supply of vapour/heat are the main factors which control the flux measured in the shallow sub-surface. However, two differences between the November 1998 and November 2004 responses give a potential insight into the exact cause of the observed variations.

Firstly, the pseudo-periodicity of the flux variations seems to be very different between the two cases. In November 1998, only one pulse was observed, with a duration of a few months. In November 2004 the duration of each pulse (Fig. 6b) was of the order of a few days.

Secondly, the total energy released in November 2004 was at least 3 times that of the November 1998 response. In November 1998, this energy could be probably provided by the boiling aquifer alone. It was not the case in November 2004, where the energy generated required increased input from the magmatic source. From these two characteristics we can assume that the causes of the November 1998 and November 2004 crises were related to changes in seismically induced permeability variations factors and magma migration, respectively.

New $\mathrm{CO}_{2}$ data for the period 1979-2006 (Granieri et al. 2006; Diliberto et al. 2005; A. Paonita, personal communication 2005) confirm the conclusion that the heat flux increase beginning in November 2004 could be ascribed to changes in input from the magmatic source. During the August 1996 thermal crisis, the $\mathrm{CO}_{2}$ concentration exceeded $18 \%$ (Fig. 8), approaching the composition of the inferred magmatic component (Granieri et al. 2006). Between August 1996 and 14 November 1998 (that is 3 days before the beginning of the seismic crisis), the value remained high $(14.5 \%)$. It then decreased to $5.6 \%$ by 1 February 1999. It remained low until 24 November 2004 when it returned to a high value (18.3\%). Before 1996, three other similarly high $\mathrm{CO}_{2}$ concentrations periods were observed: these were during 1979-81, 1985 and 1988. These periods also corresponded to moments of increasing volatile release from a magmatic source (Granieri et al. 2006).

Variations in soil-temperature measurements in a subfumarolic area, when coupled with seismic and gas data, can thus provide information on the two possible origins of changes in heat flux. Such measurements allow us to determine whether heat flux variations are a result of permeability variations in the sub-surface layer due to seismic activity or due to changes in the magmatic source conditions, which also correlate with variations of $\mathrm{CO}_{2}$ concentrations measured at the fumarole field. Distinction between these two origins could contribute to the assessment of the risk related to impending eruption in the second case.

The present conclusions are based on only one active station with continuous temperature measurements during relatively short periods spread over 7 years (and nine stations where $T_{30}$ was temporarily measured 5 times over the same period. With this deployment, flux variations were mea-

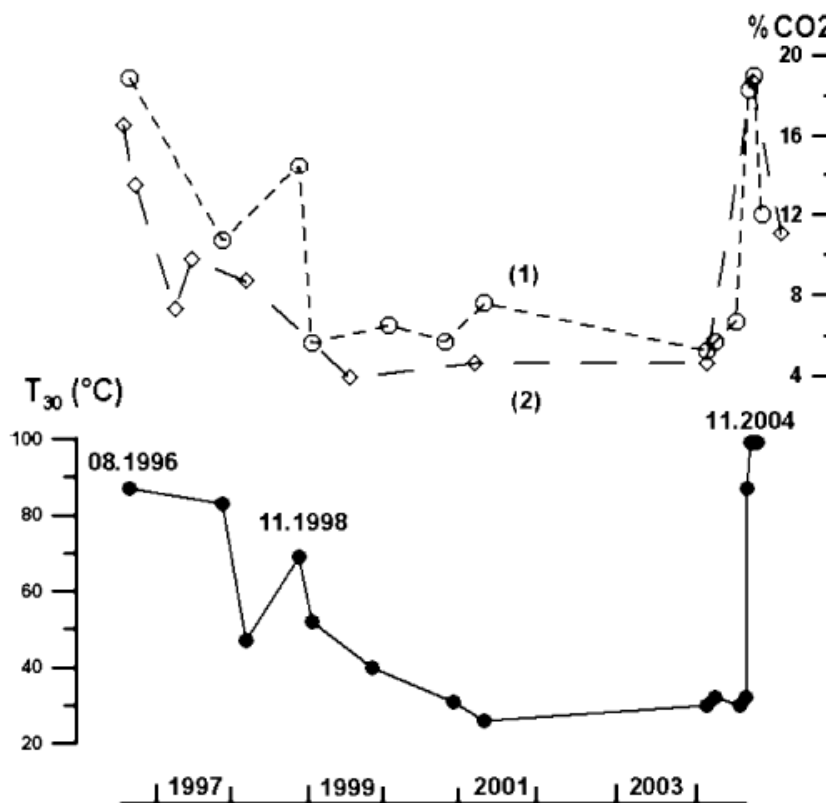

Fig. $8 T_{30}$ evolution at S4 from 1996 to April 2005 (black circles) and $\% \mathrm{CO}_{2}$ (gas/steam ratio from the high temperature fumarole field) taken from unpublished data from INGV Section of Palermo (white circles) and Granieri et al. (2006; white diamonds) 
sured at only one location ( $\mathrm{S} 4$ and the surrounding area). These current data would be much improved if ten or more continuous stations could be installed, linked by temporary profiles where $T_{30}$ would be periodically measured. In this way, it would be possible to calculate flux variations over a significant part of the Fossa crater and thus estimate not only temporal but also spatial changes in heat flux.

Acknowledgements This work was supported by EC Tekvolc contracts PL950250 and PL970584, as well as by a contract between M. Aubert and INGV, Sezione di Palermo. We warmly thank the two anonymous reviewers and Andrew Harris for their contributions which largely improved the previous manuscript. We would also like to thank J.-M. Barnagaud, S. Durand, V. Francofonte and M. Koyama for their help in the field; P. Madonia, A. Mastrolia and P. Ricobono for the improvement of equipment; S. Alparone for the communication of unpublished data regarding the seismic monitoring, the Geochemical Surveillance Functional unit, INGV Palermo, for communication of unpublished data, Frances van Wyk de Vries for correcting the English. This is IPGP contribution No. 2253.

\section{References}

Aubert M (1999) Practical evaluation of steady heat discharge from dormant active volcanoes: case study of Vulcarolo fissure (Mount Etna, Italy). J Volcanol Geotherm Res 92:413-429

Aubert M, Alparone S (2000) Hydrothermal convective flux variation related to a seismo-tectonic crisis in the Fossa of Vulcano (Italy). CR Geosci 330:603-610

Capasso G, Favara R, Francofonte S, Inguaggiato S (1999) Chemical and isotopic variations in fumarolic discharge and thermal waters at Vulcano Island (Aeolian Islands, Italy) during 1996: evidence of resumed activity. J Volcanol Geotherm Res 88:167-175

Chiodini G, Cioni R, Marini L, Panichi C (1995) Origin of fumarolic fluids of Vulcano Island, Italy and implications for volcanic surveillance. Bull Volcanol 57:99-100

Chiodini G, Granieri D, Avino R, Caliro S, Costa A (2005) Carbon dioxide diffuse degassing and estimation of heat release from volcanic and hydrothermal systems. J Geophys Res 110:B08204. DOI 10.1029/2004JB003542
Diliberto I, Finizola A, Madonia P, Aubert M (2005) Convective heat flux from hydrothermal system: first monitoring results at $\mathrm{La}$ Fossa of Vulcano. Abstract Volume of the General Assembly IAVCEI Commission on the Chemistry of Volcanic Gases-9th Gas Workshop. Palermo, Italy, May 2005

Fortran Scientific Subroutine Library (1985) Wave-3.7. Software. Fortran Scientific Subroutine Library, Peerless Engineering, Burnaby, Canada

Granieri D, Avino R, Carapezza ML, Chiodini G, Ranaldi M, Ricci T, Tarchini L (2006) Correlated increase in $\mathrm{CO}_{2}$ fumarolic content and diffuse emission from La Fossa crater (Vulcano, Italy): evidence of volcanic unrest or increasing gas release from a stationary deep magma body? Geophys Res Lett 33:L13316. DOI $10.1029 / 2006$ GL026460

Hardee HC (1982) Permeable convection above magma bodies. Tectonophysics 84:179-195

Harris AJL, Maciejewski AJH (2000) Thermal surveys of the Vulcano Fossa fumarole field 1994-1999: evidence for fumarole migration and sealing. J Volcanol Geotherm Res 102:119-147

Harris AJL, Stevenson DS (1997) Magma budgets and steady-state activity of Vulcano and Stromboli. Geophys Res Lett 24(9):1043-1046

Hill DP, Pollitz F, Newhall C (2002) Earthquake-volcano interactions. Phys Today 55(11):41-47

Italiano F, Pecoraino G, Nuccio PM (1998) Steam output from fumaroles of an active volcano: tectonic and magmatic-hydrothermal controls on the degassing system at Vulcano (Aeolian Arc). J Geophys Res 103(B12):29829-29842

McKenzie D, Brune JN (1972) Melting on fault planes during large earthquakes. Geophys J R Astr Soc 29:65-78

Montalto A (1994) Seismic signals in geothermal areas of active volcanism: a case study from "La Fossa", Vulcano (Italy). Bull Volcanol 56:220-227

Montalto A (1996) Signs of potential renewal of eruptive activity at La Fossa (Vulcano, Aeolian Islands). Bull Volcanol 57:483-492

Nuccio PM, Paonita A, Sortino F (1999) Geochemical modeling of mixing between magmatic and hydrothermal gases: the case of Vulcano Island, Italy. Earth Planet Sci Lett 167:321-333

Tabbagh A, Trezeguet D (1987) Determination of sensible heat flux in volcanic areas from ground temperature measurements along vertical profiles: the case study of Mount Etna (Sicily, Italy). J Geophys Res 92(B5):3635-3644

Ventura G, Vilardo G, Milano G, Pino NA (1999) Relationships among crustal structure, volcanism and strike-slip tectonics in the Lipari-Vulcano volcanic complex (Aeolian Islands, southern Tyrrenian sea, Italy). Phys Earth Planet Int 116:31-52

Walter TR, Amelung F (2006) Volcano-earthquake interaction at Mauna Laua volcano, Hawaii. J Geophys Res 111:B05204. DOI 10.1029/2005JB003861 